they analyzed the sequence of this gene in DNA samples from 216 patients with sporadic tumors or idiopathic pancytopenia, 109 patients with familial or multiple cancers, and 475 non-cancer controls.

A germ-line polymorphism was found (G446A), which resulted in a stop codon at position 149 of the ARLTS1 gene (Trp149Stop). This variant occurred at a similar frequency in patients with sporadic tumors and controls, but was significantly more common in those with familial cancer (odds ratio 5.7, 95\% Cl 1.3-24.8, $P=0.02)$. Transfection studies in mice then revealed that the wild-type ARLTS1gene suppressed tumor formation and induced apoptosis, whereas the G446A (Trp149Stop) variant had only a partial effect on these functions.

The researchers propose that ARLTS1 is a low-penetrance tumor-suppressor gene and that the G446A (Trp149Stop) nonsense polymorphism predisposes patients to familial cancer.

Original article Calin GA et al. (2005) Familial cancer associated with a polymorphism in ARLTS1. N Engl J Med 352: $1667-1676$

\section{Relationship between birth weight and cancer risk}

Smaller birth size, as an indicator of the fetal environment, has been linked to an increased risk of ischemic heart disease and type 2 diabetes. Conversely, a larger size at birth has been associated with increased rates of adult cancer, although few robust data are available to support this hypothesis. McCormack et al. have recently examined this question in a large cohort study.

The analysis, based on the Uppsala Birth Cohort Study, included 11,166 Swedish men and women born between 1915 and 1929 . Details of maternal age, birth order, birth weight and placental weight were recorded for all participants. Follow-up extended from 1960 to 2001 and 2,685 first primary cancers were recorded during this period.

In both sexes, higher birth weight was associated with moderately increased rates of digestive and lymphatic cancers. In women, higher birth weight was also related to an increase in the rate of breast cancer before the age of 50 years, and an inverse relationship was seen between birth weight and endometrial cancer risk. No associations were found between birth weight and cancers at other sites.

Noting that these associations were modest and only related to a few cancer sites, the authors suggest that more studies are needed to investigate pre-natal influences on cancer risk.

Original article McCormack VA et al. (2005) Birth characteristics and adult cancer incidence: Swedish cohort of over 11,000 men and women. Int J Cancer 115: 611-617

\section{Do patient preferences reduce the validity of randomized trials?}

Although a randomized controlled trial is generally accepted as the most effective way to assess clinical efficacy, patients' preferences for a particular treatment might affect the study's validity. This is particularly relevant when treatments are not blinded, and is likely to become increasingly important as patients take an ever more active interest in their management.

To assess the magnitude of any effect of patient choice on recruitment or outcomes, King and colleagues have carried out a systematic review of clinical studies that recorded patient or physician treatment preference. The selected studies followed up all participants, whether allocated to random or preference cohorts.

The results revealed that a considerable proportion of patients refused randomization because they preferred one treatment over another. In 14 of 27 studies included in the analysis, more than $50 \%$ of individuals refused randomization after having agreed to participate in the trial. Reassuringly, however, there was little bias in the characteristics of those who were randomized, and only small differences were found between the randomized and preference groups in terms of outcomes.

King et al. conclude that although recruitment to randomized trials is strongly affected by patients' preferences, neither external nor internal validity appears to suffer significantly. These findings also lend support to the use of observational studies in areas where randomization is not appropriate.

Original article King $\mathrm{M}$ et al. (2005) Impact of participant and physician intervention preferences on randomized trials: a systematic review. JAMA 293: 1089-1099 